\title{
Connection between competence, usability, environment and risk of falls in elderly adults
}

\author{
José Alex Leiva-Caro ${ }^{1}$ \\ Bertha Cecilia Salazar-González² \\ Esther Carlota Gallegos-Cabriales ${ }^{2}$ \\ Marco Vinicio Gómez-Meza ${ }^{3}$ \\ Kathleen F. Hunter ${ }^{4}$
}

Objective: to determine connections between competence, usability, environment and risk of falls in elderly adults. Method: correlational descriptive study, 123 elderly adults, both male and female, aged 70 years and older were included. Data was collected via the Tinetti Scale, CESD-7 Scale, Montreal Cognitive Assessment, Usability Questionnaire on Housing and Housing Enabler; and sociodemographic and health background certificate data. For data analysis, descriptive and inferential statistics were used, multivariate linear and logistic regression models were adjusted. Results: $42.0 \%$ of the elderly adults had presented with falls, with a higher prevalence in women, and in the group of 70-75 years. The physical environment of the house, gait, and usability were set as risk factors for falls. A negative relationship between usability and depressive symptoms, cognitive health, balance, gait, the social and physical environment was found, $p<0.05$; and a strong positive correlation between walking and balance, $p<0.05$. Conclusion: this study helps to better understand the phenomenon of falling, to find a connection between usability with the risk of falls, and other variables.

Descriptors: Risk Factors; Environment; Accidental Falls; Aged.

\footnotetext{
${ }^{1} \mathrm{PhD}$, Professor, Departamento de Enfermería, Facultad Ciencias de la Salud y de los Alimentos, Universidad del Bío-Bío, Chillán, Chile. 2 PhD, Professor, Facultad de Enfermería, Universidad Autónoma de Nuevo León, San Nicolás de los Garza, NL, Mexico.

3 PhD, Professor, Facultad de Economía, Universidad Autónoma de Nuevo León, San Nicolás de los Garza, NL, Mexico.

${ }^{4}$ PhD, Associate Professor, Faculty of Nursing, University of Alberta, Edmonton, AB, Canada.
}

Corresponding Author: Dr. José Alex Leiva-Caro Universidad del Bío-Bío. Facultad Ciencias de la Salud y de los Alimentos Departamento de Enfermería CP: 3780000 , Chillán, Chile E-mail: jleiva@ubiobio.cl
Copyright () 2015 Revista Latino-Americana de Enfermagem This is an Open Access article distributed under the terms of the Creative Commons Attribution Non-Commercial License (CC BY-NC).

This license lets others distribute, remix, tweak, and build upon your work non-commercially, and although their new works must also acknowledge you and be non-commercial, they don't have to license their derivative works on the same terms. 


\section{Introduction}

Increased life expectancy and declining fertility have caused a demographic shift in Mexico and in the world, thus there has been an increase in the elderly adult population (EA). Consequently, an increased demand for health services is expected by this population, where falls take up a high percentage of the services due to serious repercussions, considering that it has currently become a public health problem(1).

Falls are ranked as the second highest comorbidity in elderly Mexican adults, with $42.9 \%{ }^{(2)}$; in Santiago de Chile, the prevalence of falls is $34.0 \%$, slightly higher than Mexico City (33.5\%), but above Sao Paulo (29.0\%) and Montevideo $(27.0 \%)^{(3)}$. Falls can cause different effects, ranging from minor injuries to fractures ${ }^{(1)}$ and death $^{(4)}$. These consequences impact health agencies by increasing the use of personal and material benefits derived from medical care and nursing resources; therefore, falls are considered the highest injury cost among the $\mathrm{EA}^{(5)}$.

The etiology of falls is multicausal(6), with individual factors such as drug use, fear of falling, deterioration in gait, decreased activities of daily living(1) or environmental factors and hazards in the home ${ }^{(7)}$, including loose rugs, differences in levels and slippery floors. Now, we propose that rather than identifying the causes, identifying risk factors is most useful(6), because the cause of a fall is a past event, which cannot be influenced. While environmental hazards are common in the homes of EA with or without disabilities ${ }^{(8)}$, their role as risk factors for falls is unclear, since some household characteristics have been identified as factors contributing to half the number of falls; although other studies on this phenomenon do not agree ${ }^{(8)}$. It has been suggested that the lack of clarity in the literature in relation to environmental factors and falls can be attributed to the fact that the person-environment interaction has not been assessed ${ }^{(9)}$. On the one hand, individual characteristics are observed in elderly adults, in the other hand the characteristics of his immediate environment are observed. There is also an interaction between them. Other authors support the assessment of the interaction between the physical abilities of a person and their exposure to environmental stress factors $^{(10)}$. For such reasons, it is necessary to consider the characteristics of the individual, his immediate environment, and the interaction between them to determine the risks of falling, and consequently, propose measures to prevent further falls due to the same factor.
In the literature review, studies that somehow reflect the interaction of EA with the environment and falls can be found ${ }^{(9,11)}$; however, they have failed to establish a conclusive link between EA and the environment with the risk of falling. Added to this, these studies have weaknesses, failing to include important individual variables such as drug consumption (type / quantity) and fear of falling, among others, which according to a systematic review, are widely reported in studies on falls ${ }^{(1)}$. For these reasons, it was proposed to study how elderly adults act and interact with the environment in which they live and how they perceive it; so the personal component was studied in terms of competence (functional capacity, cognitive health, depressive symptoms), physical and social environment (according to characteristics of the house and the family component, i.e., numbers of people with whom they live), and the interaction of the EA with the environment, which is termed as usability.

The concept of usability implies that a person should be able to use the resources of the environment in conditions of equality with others, it corresponds to the appreciation of EA regarding the extent to which they can perform activities in the house, and includes the activity component; according to the authors this is a measure of effectiveness, efficiency and satisfaction in the use of resources ${ }^{(12)}$. It also reflects the personal motivation, adaptive skills, preferences and the need for activity, and the subjective evaluation of the environmental demands(13).

This study was guided by the Ecological Model of Competence ${ }^{(14)}$, which focuses on both the competences of the individual and the environment. First, one considers how the reduction of individual proficiency affects the results of the individual to interact with the environmental pressure; and second, how the environmental pressure can affect individual proficiency, resulting in an adaptive or maladaptive behavior. This study aims to determine the connection of competence, usability and the environment with the risk of falls in the EA.

\section{Method}

The study was descriptive, correlational, and was conducted in the city of Monterrey, Nuevo Leon, Mexico. The sample included EA who intended to obtain or renew their elderly adult ID from the Instituto Nacional de las Personas Mayores (INAPAM). The sample size was calculated for a significance level of 0.10 , success ratio of 
0.30 , odds ratio $1.9,0.2$ coefficient of determination, and power of $90.0 \%$, which resulted in $\mathrm{n}=123$ individuals. Elderly adults aged 70 years and above who voluntarily agreed to participate were included; we excluded those who could not hear the voice of the interviewer, or if the interviewer did not understand what they said (inaudible voice, inarticulate), those who expressed the need for assistance from a third person or who were blind were excluded. A trained research assistant invited them to participate, explained the purpose of the study, and confirmed the inclusion / exclusion criteria; then the date and time of the visit to the home address by the main researcher (MR) was agreed. The MR phoned the day before the visit to confirm the appointment and verify the inclusion / exclusion criteria, all of which were ratified for the last time during the visit. The MR conducted the data collection from February to June of 2013, making use of five instruments and data from one certificate of sociodemographic and health background. According to the Ecological Model of Competence (14), the following variables and measuring instruments were considered competences: functional capacity in terms of gait and balance (modified Tinetti Scale(15)), depressive symptoms (CESD -7 Scale ${ }^{(16)}$ ), and cognitive health (Montreal Cognitive Assessment $\left.{ }^{(17)}\right)$; elderly adult interaction with the environment was represented with usability (Housing Usability Questionnaire(18), regarding the environment, the physical environment was considered, based on environmental barriers (Housing Enabler instrument(19) ), and social environment, corresponding to the number of people living with the elderly adult (information contained in the document with the certificate data). The instruments used are described below.

The modified Tinnetti Scale ${ }^{(15)}$ assesses gait and balance, both scores are useful in establishing the risk of falls. The scale was used to assess the functional capacity of the elderly adult, and classify it as a normal (score greater than 24 points), adapted (score between 19 and 24 points) and abnormal (score less than 19 points). For purposes of the correlations, the maximum raw score for balance (16 points) was converted to an index of 0 to 100 , where a lower score represents a greater balance disorder. Likewise, the maximum raw score for gait (12 points) was converted to an index of 0 to 100 , where a lower score represents greater gait disorder. The CESD-7 Scale ${ }^{(16)}$ assesses the presence of depressive symptoms during the week prior to the assessment; in terms of frequency, it consists of 7 Likert-like items; instrument raw values are between 0 and 21 points, which were converted to an index of 0-100, where a higher score represents greater presence of depressive symptoms.

The Montreal Cognitive Assessment (MoCA)(17) assesses mild cognitive dysfunctions. It assesses seven skills (visuospatial / executive, identification, attention, language, abstraction, deferred memory, and orientation level), then the scored points are summed, a score equal to or above 26 corresponds to a normal individual, and a lower score was classified with mild cognitive impairment. For purposes of correlations, scores were transformed to an index from 0 to 100 points, where a higher score represents less cognitive impairment. The Housing Usability Questionnaire(18), assesses the usability of the immediate internal and external resources of the house (e.g., kitchen, living room, patio), and includes the corridors. There are open-ended questions and some others have possible answers with seven points ( 1 to 7 ), where 1 represents the worst or lowest alternative for the subject and 7 represents the best or highest alternative. Raw values for the instrument were converted to an index from 0 to 100, with higher scores representing better or greater usability perceived by the elderly adult. The Housing Enabler(19) instrument allowed us to assess the physical environment of the house according to the presence or absence of barriers. The score of this instrument was converted to an index from 0 to 100 points, with higher scores representing greater magnitude of environmental barriers in the house and lower scores representing lower magnitude of environmental barriers.

The certificate of general data designed for the study yielded information about age, gender, education, number of people with whom the EA lives, time living in the house (in years); presence of falls six months before assessment and fear of a fall was inquired about, a dichotomous variable with a yes or no answer. It is noteworthy that, according to the proposed results in the Ecological Model(14) as a product of the interaction between the competence and environmental pressure, adaptive behavior corresponded to the elderly adult with "no risk of falls / no falls" and in non-adaptive behavior, to the elderly adult with "risk of falls / with falls." A decrease in far vision was assessed via a dichotomous (yes or no) Snellen Test; the number of diagnosed diseases and the number of drugs taken daily was contrasted with the health or family control document.

Data analysis was conducted using the Statistical Package for the Social Sciences (SPSS), version 
16.0 for Mac OSX. Descriptive statistics (measures of central tendency, variability, frequencies and percentages) and inferential statistics were used. The normality test of Kolmogorov-Smirnov was used with Lilliefors' correction according to the results; the Spearman hierarchies were used for the correlation coefficient. Multivariate models of multiple linear regression (MANOVA) and logistic regression were adjusted. Both correlation and regression models were useful for testing the of Ecological Competence Model concept relationship ${ }^{(14)}$, which states that the person is affected when his individual competence is diminished and he faces environmental pressure. This pressure, in turn, can affect the competence of the individual resulting in an adaptive or nonadaptive behavior. This study aims to determine the connection of competence, usability (interaction) and the environment with the risk of falls in the EA (nonadaptive behavior).

The study was approved by the Committees on Ethics and Research of the Facultad de Enfermería de la Universidad Autónoma de Nuevo León (Registry number: FAEN-D-912), and with the authorization of INAPAM to invite the EA to participate.

\section{Results}

A total of 123 individuals and their homes were assessed, all of them residents of six municipalities in the metropolitan area of Monterrey. Sixty-four percent were women, the mean age was 77.5 years (SD 7.03 years), and $50.0 \%$ were found in the group of 70-75 years. The average length of schooling was 6.4 years (SD \pm 5.09 ). Only $16.3 \%$ reported living alone, and the average time living in the house was 31 years ( $S D \pm 15.9)$. For diseases, $89.0 \%$ reported having a health problem; $84.0 \%$ used medications, the highest daily consumption was between three and four medications (36.6\%), and $81 \%$ had impaired distance vision. According to the Ecological Model ${ }^{(14)}$ on the non-adaptive behavior, $42.0 \%$ had experienced at least one fall within the six months prior to the assessment; the average age of those who had fallen was 78 years; $30 \%$ reported fear of falling. The highest prevalence by age (Table 1 ) occurred in the group of $70-75$ years, and by gender in women.
Table 1 - Prevalence of falls in the elderly adults by gender and age. Monterrey, NL, México, $2013(n=123)$

\begin{tabular}{lcccc}
\hline \multirow{2}{*}{ Variable } & \multicolumn{2}{c}{ Falls } & \multicolumn{2}{c}{ No falls } \\
\cline { 2 - 5 } & $\mathbf{n}$ & $\%$ & $\mathbf{n}$ & $\%$ \\
\hline $\begin{array}{l}\text { Gender } \\
\text { Female }\end{array}$ & 32 & 61.5 & 47 & 66.2 \\
Male & 20 & 38.5 & 24 & 33.8 \\
Age & & & & \\
$70-75$ & 26 & 50.0 & 36 & 50.7 \\
$76-80$ & 7 & 13.5 & 14 & 19.7 \\
$81-85$ & 11 & 21.1 & 12 & 16.9 \\
86 or older & 8 & 15.4 & 9 & 12.7 \\
\hline
\end{tabular}

In terms of the Ecological Model(14) to review the competence of the elderly adult, in the Tinetti Scale (gait and balance), 35.8\% had normal functional capacity, $31.7 \%$ adaptive abnormal functional capacity, and $32.5 \%$ abnormal functional capacity; $82.9 \%$ had mild cognitive impairment and $40.7 \%$ had higher depressive symptoms. Regarding the interaction, $49.5 \%$ had high usability, and in terms of physical environment, $49.5 \%$ reported a greater number of barriers in the home. With no breakpoints in the instrument, the index values of the CESD-7 above the mean (30) were considered, to characterize the elderly adults with more depressive symptoms, and fewer symptoms those who had scores below the mean. For usability (mean =94) and physical environment of the apartment (mean $=41$ ), the figures above the mean of the indexes represented more usability and presence of barriers to housing respectively. Regarding the risk of falls, which corresponds to a non-adaptive behavior according to the Ecological Model (14), it was found that the physical environment of the housing is directly related to the risk of falling, ( $B$ $=-0311, \mathrm{SE}=0.183,95 \% \mathrm{CI}[0.99-1.05], \mathrm{p}=$ 0.08). In turn, the overall regression model found that usability (interaction) is related to the risk of falling $(B=-0.052, S E=0.034,95 \% C I[0.74$ to $0.15], p=0.08)$. Finally, with regard to the risk of falling, according to the competence and physical environment of the housing (Table 2), it was found that gait is the only significant risk factor present for fall risk. 
Table 2 - Risk of falls in the elderly adults according to competence and physical environment. Monterrey, NL, México, $2013(n=123)$

\begin{tabular}{|c|c|c|c|c|c|}
\hline \multirow{2}{*}{ Variables } & \multirow{2}{*}{ Coefficient B } & \multirow{2}{*}{ Standard Error } & \multirow{2}{*}{$\mathbf{p}^{*}$} & \multicolumn{2}{|c|}{$\mathrm{Cl} 95 \%$} \\
\hline & & & & Lower Limit & Upper Limit \\
\hline Physical Environment & 0.020 & 0.016 & 0.201 & 0.989 & 1.052 \\
\hline Depressive symptoms & 0.003 & 0.009 & 0.692 & 0.987 & 1.020 \\
\hline Balance & 0.023 & 0.017 & 0.171 & 0.990 & 1.058 \\
\hline Gait & -0.032 & 0.014 & $0.019^{\dagger}$ & 0.942 & 0.995 \\
\hline Cognitive Health & 0.008 & 0.011 & 0.459 & 0.987 & 1.029 \\
\hline Constant & -1.196 & 1.131 & 0.290 & & \\
\hline
\end{tabular}

Cox and Snell $R^{2}=0.069 ;$ Nagelkerke $R^{2}=0.092$.

* Significance level

+ Significant risk factor $p<0.05$

To determine the connection between variables (Table 3), a bivariate analysis was performed; in that sense the classification scheme of correlation coefficients presented by Morton (20) is useful if you want to analyze the practical significance of these coefficients. Consistent with its classification, the correlation matrix of Table 3 has 21 negligible coefficients (less than 0.0 - 0.2), 12 weak (< $0.2-0.5)$, two moderate (0.5 $<0.8)$ and one strong $(0.8-1.0)$. Among the significant correlations, the connection between usability with social environment, depressive symptoms, cognitive health, balance, gait and physical environment are highlighted. A strong correlation occurs between balance and gait, with a positive and highly significant result $(r=0.816)$.

A model of multivariate linear regression analysis was adjusted for the effect of gender, age, number of people living with an EA, number of medications, fear of falling, and decreased distance vision on competence (depressive symptoms, balance, gait and cognitive health), usability, and physical environment of the house; the Wilks Lambda $(\Lambda)$ was used as statistical test (Table 4). It was found that age alone was shown to have a significant effect on the six dependent variables. The selection of variables with the "backwards" technique required five additional adjustments of multivariate linear regression models, where the order of elimination of variables was: (1) fear of a fall, (2) number of medications taken a day, (3) gender, (4) decreased distance vision, and (5) number of people the EA lived with. The final model included only the age of the elderly adult, $(L=0.533$, $F(6,116)=16.95 ; p<0.001)$.

Table 3 - Spearman correlation matrix. Monterrey, NL, México, 2013 ( $n=123$ )

\begin{tabular}{|c|c|c|c|c|c|c|c|c|c|}
\hline Variable & $\mathrm{X} 1$ & $\mathrm{X} 2$ & $\mathrm{X} 3$ & X4 & $\times 5$ & $\mathrm{X} 6$ & $\mathrm{X} 7$ & $\mathrm{X} 8$ & X9 \\
\hline X1. Age & $7.038^{*}$ & $0.662^{\dagger}$ & $0.069^{\dagger}$ & $0.744^{\dagger}$ & $0.001^{\dagger \ddagger}$ & $0.001^{\dagger \ddagger}$ & $0.001^{\dagger \ddagger}$ & $0.155^{\dagger}$ & $0.378^{\dagger}$ \\
\hline X2. Social E. & $0.040^{\S}$ & $1.727^{*}$ & $0.686^{\dagger}$ & $0.977^{\dagger}$ & $0.173^{\dagger}$ & $0.284^{\dagger}$ & $0.579^{\dagger}$ & $0.005^{\dagger \neq}$ & $0.293^{\dagger}$ \\
\hline X3. Number of medications & $0.164^{\S}$ & $-0.037^{\S}$ & $2.240^{*}$ & $0.762^{\dagger}$ & $0.989^{\dagger}$ & $0.032^{\dagger \ddagger}$ & $0.039^{\dagger \ddagger}$ & $0.454^{\dagger}$ & $0.848^{\dagger}$ \\
\hline X4. Depressive symptoms & $0.030^{\S}$ & $-0.003^{\S}$ & $0.028^{\S}$ & $24.688^{*}$ & $0.001^{\dagger \ddagger}$ & $0.001^{\text {t‡ }}$ & $0.002^{\dagger \ddagger}$ & $0.001^{\dagger \ddagger}$ & $0.119^{\dagger}$ \\
\hline X5. Cognitive health & $0.374^{\S}$ & $-0.124^{\S}$ & $0.001^{\S}$ & $-0.439 \S$ & $22.090^{*}$ & $0.001^{\dagger \ddagger}$ & $0.001^{\dagger \ddagger}$ & $0.002^{\dagger \neq}$ & $0.048^{\dagger \neq}$ \\
\hline X6. Balance & $0.581^{\S}$ & $-0.097^{\S}$ & $-0.194^{\S}$ & $-0.353^{\S}$ & $0.453^{\S}$ & $23.263^{*}$ & $0.001^{\dagger \ddagger}$ & $0.001^{\dagger \ddagger}$ & $0.786^{\dagger}$ \\
\hline X7. Gait & $0.624^{\S}$ & $-0.050^{\S}$ & $-0.186^{\S}$ & $-0.276^{\S}$ & $0.486^{\S}$ & $0.816^{\S}$ & $29.521^{*}$ & $0.001^{\dagger \neq}$ & $0.667^{\dagger}$ \\
\hline X8. Usability & $0.129 \S$ & $-0.250 \S$ & $0.068^{\S}$ & $-0.331^{\S}$ & $0.271^{\S}$ & $0.321^{\S}$ & $0.318^{\S}$ & $5.937^{*}$ & $0.001^{\text {t‡ }}$ \\
\hline X9. Physical E. & $0.080^{\S}$ & $0.096 \S$ & $-0.017^{\S}$ & $0.141^{\S}$ & $-0.178^{\S}$ & $-0.025^{\S}$ & $0.039^{\S}$ & $-0.304^{\S}$ & $12.691^{*}$ \\
\hline Mean & 77.5 & 2.15 & 3.07 & 29.7 & 58.0 & 73.3 & 68.5 & 93.7 & 40.5 \\
\hline Median & 75.0 & 2.00 & 3.00 & 23.8 & 56.6 & 81.2 & 75.0 & 92.6 & 40.0 \\
\hline Minimum Value & 70 & 0 & 0 & 0 & 3.33 & 6.25 & 0 & 60.71 & 7.5 \\
\hline Maximum Value & 100 & 7 & 10 & 100 & 100 & 100 & 100 & 100 & 75 \\
\hline
\end{tabular}

* Standard deviation

+ Bilateral $p$ values

\# Significative correlation $p<0.05$

$\S$ Spearman Correlation Coefficient 
Table 4 - Statistical contrasts resulting in adjusting the multivariate model of multiple linear regression, considering depressive symptoms, balance, gait, cognitive health, usability and physical environment of the house as dependent variables. Monterrey, $\mathrm{NL}$, México, $2013(n=123)$

\begin{tabular}{lccc}
\hline \multicolumn{1}{c}{ Effect } & $\boldsymbol{\Lambda}$ & $\boldsymbol{F}^{*}$ & $\boldsymbol{p}^{\dagger}$ \\
\hline Intercept & 0.815 & 81.683 & 0.001 \\
Gender & 0.948 & 1.024 & 0.414 \\
Age (years) & 0.602 & 12.224 & $0.001^{\ddagger}$ \\
Fear of falling & 0.981 & 0.353 & 0.907 \\
Number of people sharing the & 0.920 & 1.598 & 0.154 \\
household & & & \\
Number of drugs & 0.938 & 1.220 & 0.301 \\
Decrease in distance vision & 0.933 & 1.320 & 0.254 \\
\hline
\end{tabular}

* F Statistical with 6 and 111 degrees of range, for the hypothesis and error respectively

+ Significance Level

₹ Significant effect $p<0.05$

\section{Discussion}

The study responds to the objective to find a connection between the variables of interest with the risk of falls, and reaffirms gait as a risk factor for falls in EA reported in other studies ${ }^{(1,6)}$. The mean age of those who had fallen was 78 years, which is consistent with another study in the Mexican population(21); regarding gender, the largest proportion of falls occurred in females, a fact that is documented worldwide(22-23).

The fact that the physical environment of the house is directly related to the risk of falling is consistent with a meta-analysis in which it was stated that the dangers in the home increase the risk of falling(7). While the number of housing barriers was low, it is important to consider the type of housing barriers and the personal characteristics of the EA in terms of competence, since the fall occurred when the EA interacted with the environment. Now, in accordance with the Ecological Model(14), a high environmental pressure on an EA with reduced competence would lead to maladaptive behavior, in this case a fall. Among the high prevalence competences of an EA that appeared reduced in this study were: impaired distance vision, health problems, cognitive impairment and abnormal gait, which undoubtedly are factors that can affect the EA-environment interaction and the resulting level of adaptation. It is also important to consider the time living in the house (in our study, the mean time living in the household was 31 years), as it shows that being exposed for too long to the dangers of the environment does not mean that they can overcome them safely, or they perceive them as normal and not as a risk for falls. This could also be related to the presence of cognitive impairment and gait disturbance. Moreover, this is further supported on one hand by the positive connection that was found between cognitive health with balance and gait, and on the other hand by the negative connection between cognitive health and the physical environment. With regard to the connection between cognition and the physical environment, which suggests that the number of environmental barriers increases with cognitive impairment, no similar reports have been found. Therefore it is proposed to consider when cognition or the environment are evaluated, as well as interventions that seek to improve the interaction of EA with the physical environment. It is therefore necessary to consider both the number of housing barriers in future studies, as well as to determine whether the barrier increased the risk of falls for EA, such as the presence of stairs, loose wires, lack of handrails, house lighting, among others.

The study found that usability, i.e. the interaction of EA with their immediate environment, is directly related to the risk of falling, which is a result that defers to those reported worldwide ${ }^{(9)}$. Our result is the first to find a link between usability and the risk of falling, which means that elderly adults with low usability are at greater risk of falling. The low usability suggests that an EA with less use of environmental resources may have muscle disuse atrophy and limit his interaction with the environment. In addition, this could be aggravated by the presence of other factors, such as cognitive impairment and balance. This is supported by other findings of the study, such as the positive connection of usability with gait and balance, suggesting that an EA with high usability will have a normal functional capacity, or close to it, and will encourage the safe interaction with the environment and thus reduce the risk of falls. Also, a negative connection between usability and depressive symptoms and cognitive health was found. This suggests that in EA with depressive or cognition problems, usability decreases and therefore so do the interactions. These results support what is being proposed in the Ecological Model(14), which is that aging diminishes competence (depressive symptoms, cognitive impairment) and consequently, the individual can withdraw himself or try to compensate by resorting to so-called primitive cognitive styles, in which the person represses himself and opts for simplicity to reduce tension between the environment and his capabilities. Either option (withdrawal or compensation through repression) makes him more vulnerable because it somehow leads 
to inactivity and atrophy, affecting usability with the consequent risk of adverse events such as falls.

The findings on the connection of usability with other variables, and as a risk factor for falls, while it may be debatable, allows us to first focus on a better understanding of the phenomenon of falling, and second, suggests considering the perception of the $E A$ on the use of the resources of the environment as an important issue to study the risk of falling, which certainly requires further study. Commonly, studies are conducted under researcher assessment without incorporating the valuation or perception that the EA has of the environment in which he lives, and how this may affect his activities. Furthermore, the results suggest the need to continue conducting studies on usability and its connection to falls with a higher number of participants, using randomized selection and carried out longitudinally. On the other hand, it leads to reshaping of interventions for the risk of falling, as it is suggested to incorporate usability as a central component in prevention activities.

We proposed to determine the risk of falls according to the competence and the physical environment of the house of the EA; gait was determined to be a significant risk factor for falls, which is well documented at an international level(1,6,24). The Ecological Competence Model(14) suggests that reduced competence is often concomitant of aging, and leads the individual to a state of vulnerability, which is supported by our result in establishing gait disturbance and risk of falls in the EA.

With regard to cognitive impairment and depressive symptoms, our results yielded no relationship to the risk of falling. This differs from other studies in which cognitive impairment ${ }^{(25)}$ and depressive symptoms ${ }^{(3,26)}$ have been reported as risk factors for falls in EA. In the matter of cognitive impairment, it is likely that this inconsistency is due to individual factors and the instrument with which the cognitive health of the EA was assessed. Another factor is how long the EA has been living with these problems. This may play a significant role in his adaptation to environmental barriers; the same situation can happen with the presence of depressive symptoms.

The study also found a negative connection between usability and the physical environment; suggesting a greater presence of environmental barriers, decreasing EA usability. Our data are consistent with those reported in another study of usability ${ }^{(13)}$. It should be noted that the usability variable is assessed through questions and environmental barriers through observation, the same strategy used in the cited studies ${ }^{(9,13)}$. The result shows that the barriers of the environment must be considered beyond the risk of falls. That is, the reduction of usability means that the use of environmental resources decreases, which can lead the EA to inactivity and thereby adversely affect the competences, which becomes a vicious cycle, and that viewed within the Ecological Model ${ }^{(14)}$, would lead to non-adaptive EA behavior. This must be dealt with by strategies aimed at reducing the number of environmental barriers to promote usability, which according to the Ecological Model(14) would correspond to an active strategy focused on the environment, which is possible only when the demand for this is within the range of possible adaptation, i.e. the resources are there. One strategy may be to install handrails on stairs, which must be implemented early and not when the EA is affected by the environment or other components that can magnify the consequences, such as decreased vision, gait disturbance or cognitive impairment. The idea is to raise awareness and stimulate the EA to be part of preventive activities to improve usability. It is noteworthy that the authors of the Ecological Model(14) proposed that instead of changing the environment or changing the place of the individual, it is possible to intervene to increase the level of competence; but also maintain that the environment can be more flexible than individual competition.

On the other hand, a negative connection between the social environment and usability was found. This suggests that a greater number of people who cohabit with the EA (two or more people) decreases usability. This result differs from another study ${ }^{(13)}$, in which negative connection was found between living alone and usability inside and outside of the house, but there was no connection between cohabitants and usability in general. Therefore, it becomes an important finding. Our results suggest that family, caregivers, or friends may be exerting some actions leading to decreased usability. These can be due to overprotectiveness or help given in situations in which it is not justified (dressing, feeding, bathing), which can affect motivation to perform these activities on one's own and thus lead to lower usability and competences (physical, cognitive, psychological, etc.), causing an induced dependency in the EA.

The result of this study is consistent with the proposal in the Ecological Model(14) when referring to the pressure of the environment; based on Murray, the authors argue that the forces or stresses on the environment, in conjunction with the individual's needs, generate a response. In our study, the largest number 
of people living with the EA (social environment) can decrease the environmental pressure when performing the tasks incumbent to the EA, thus meeting the needs and reducing stimuli negatively affecting usability (interactions).

Regarding the multivariate contrast, seeking to know the contribution of gender, age, number of people living with the EA, number of medications, fear of falling, and decreased distance vision on competence, usability and physical environment, the result showed that only age contributes in a significant manner. That is, the increase in age can affect competence (gait, balance, cognitive health, and depressive symptoms), interaction (usability) and the physical environment (presence of barriers in house). The Ecological Model(14) posits that pressure from the environment, needs and skills of the individual fluctuate over time, which is supported by our results, considering age as a factor influencing competence, usability and physical environment. As mentioned before, the authors of the Model argue that a reduced competence is often concomitant of aging. This is also supported by the findings in this study. This is exemplified by the negative connection that has been documented between age and cognitive health, gait and balance, the negative connection between depressive symptoms and cognitive health and balance, a finding consistent with other authors, as well as with the strong positive correlation between gait and balance, supported by the literature results. Therefore, the effect of age can be attributed to aging at the level of organs and systems, a process that is characterized by a decrease in functional competence. Health issues and medication consumption must also be taken into account, both considered to be risk factors of falling in the $E A^{(1)}$, and in our study it was associated negatively with gait and balance. That is, the higher the consumption of medications, the more the detriment to gait and balance. In short, the EA is incapacitated to a greater or lesser degree depending on his competences for an active and secure relationship with the environment, such as to overcome or reduce environmental barriers, which is supported by the Ecological Model(14). Therefore, the age beyond its meaning of time, becomes a relevant factor in the field of EA health, which according to it must be attentive to the level of competence, usability and housing environment. This makes it possible to implement strategies for the EA, depending on his age, in order to reduce personal risk and environment.

\section{Conclusions}

The study contributes data allowing a better understanding of the phenomenon of falling, finding a direct connection between usability and the risk of falling. In turn, it provides new information about usability and its relationship with the social environment, depressive symptoms, cognitive health, balance, gait and physical environment. It is considered important to encourage EA in usability or greater interaction with his environment and to study its implications on falls. These results confirm those reported in other studies of risks of falls, in terms of abnormal gait as a significant factor; it also provides information on the connection between age and gait, balance, depressive symptoms and environmental barriers. Regarding the Ecological Competence Model, on one side it was useful to guide the study and explain how the decrease of individual competence affects the results of the individual to interact with environmental pressure, and secondly, how the environmental pressure can affect the competence of the individual resulting in an adaptive or non- adaptive behavior in terms of falls or risk of falling. The model also allowed the explanation of the interaction of the EA in terms of usability, and considering adapting or not adapting as a result which determines the risk of falls or fall for the EA in his usual way of life (community).

For nursing, certainly the results are relevant in that they provide an area of opportunity for the development of research on falls in the EA. This information can be useful to improve the interventions in the prevention of falls in EA and rehabilitation interventions in other age groups, and allows us to address this issue comprehensively (competition, usability and environment). On the other hand, it is inconsistent with the literature on risk factors for falls, such as cognitive impairment and depressive symptoms, which were not shown to be significant in this population of EA. This shows that the phenomenon of falling, despite having been the subject of study for some time, requires further deepening of some variables or of how they are assessed. We conclude that usability is useful to determine how the EA uses the resources of the environment, as a parameter to determine the health condition of the EA, and as a risk factor for falls. As for limitations, threats to external validity included the selection of participants from all seniors who attended the INAPAM in the Municipality of Monterrey to register or renew their certificate without random selection. For this reason, the data may not be generalizable. 


\section{References}

1. Kwan MM, Close JC, Wong AK, Lord SR. Falls incidence, risk factors, and consequences in Chinese older people: a systematic review. J Am Geriatr Soc. 2011;59(3):536-43.

2. Barrantes-Monge M, García-Mayo EJ, GutiérrezRobledo LM, Miguel-Jaimes A. Dependencia funcional y enfermedades crónicas en ancianos mexicanos. Salud Pública Mex. 2007;49(4):459-66.

3. Reyes-Ortiz CA, Al Snih S, Markides KS. Falls among elderly persons in Latin America and the Caribbean and among elderly Mexican-Americans. Rev Panam Salud Publica. 2005; 17(5-6):362-9.

4. Chisholm KM, Harruff RC. Elderly deaths due to groundlevel falls. Am J Forensic Med Pathol. 2010;31(4):350-4. 5. Davis JC, Robertson MC, Ashe MC, Liu-Ambrose T, Khan KM, Marra CA. International comparison of cost of falls in older adults living in the community: a systematic review. Osteoporos Int. 2010;21(8):1295-306.

6. Rubenstein LZ. Falls in older people: epidemiology, risk factors and strategies for prevention. Age Ageing. 2006;35(2):37-41.

7. Letts L, Moreland J, Richardson J, Coman L, Edwards M, Ginis KM, et al. The physical environment as a fall risk factor in older adults: systematic review and metaanalysis of cross-sectional and cohort studies. Aust Occup Ther J. 2010;57(1):51-64.

8. Gitlin LN. Conducting research on home environments: lessons learned and new directions. Gerontologist. 2003;43(5):628-37.

9. Iwarsson S, Horstmann V, Carlsson G, Oswald F, Wahl HW. Person-environment fit predicts falls in older adults better than the consideration of environmental hazards only. Clin Rehabil. 2009;23(6):558-67.

10. Lord SR, Menz, HB, Sherrington C. Home environment risk factors for falls in older people and the efficacy of home modifications. Age Ageing. 2006;35(2):ii55-ii59.

11. Hill EE, Nguyen TH, Shaha M, Wenzel JA, DeForge $B R$, Spellbring AM. Person-environment interactions contributing to nursing home resident falls. Res Gerontol Nurs. 2009;2(4):287-96.

12. Iwarsson S, Ståhl A. Accesibility, usability and universal design-positioning and definition of concepts describing person-environment relationships. Disabil Rehabil. 2003; 25(2):57-66.
13. Fänge A, Iwarsson S. Accessibility and usability in housing: construct validity and implications for research and practice. Disabil Rehabil. 2003;25(23):1316-25.

14. Lawton MP, Nahemow L. Ecology and the aging process. In: Eisdorfer C, Lawton MP, editors. Psychology of adult development and aging. Washington (DC): American Psychological Association; 1973. p. 619-74. 15. Rubenstein LZ. Instrumentos de evaluación. En: Abrams WB, Berkow R. editores. Manual Merck de Geriatría. Barcelona: Ed Doyma; 1992. p. 1251-63.

16. Herrero J, Gracia E. Una medida breve de la sintomatología depresiva (CESD-7). Salud Mental. 2007;30(5):40-6.

17. Nasreddine ZS, Phillips NA, Bédirian V, Charbonneau $S$, Whitehead V, Collin I, et al. The Montreal Cognitive Assessment, MoCA: a brief screening tool for mild cognitive impairment. J Am Geriatr Soc. 2005;53(4):695-9.

18. Fänge $A$, Iwarsson S. Physical housing environment: development of a self-assessment instrument. Can J Occup Ther. 1999;66(5):250-60.

19. Iwarsson S, Slaug B. Housing Enabler: A method for rating/screening and analysing, accessibility problems in housing. Manual for the complete instrument and screening tool. 2.ed. Sverige (SW): Lund \& Staffanstorp, Veten \& Skapen HB och Slaug Enabling Development; 2010. 113 p.

20. Morton RF, Hebel JR, McCarter RJ. A Study Guide to Epidemiology and Biostatistics, 4.ed. Gaithersburg, MD: Aspen Publishers; 1996. p. 92-7.

21. Manrique-Espinoza B, Salinas-Rodríguez A, MorenoTamayo K, Téllez-Rojo M. Prevalencia de dependencia funcional y su asociación con caídas en una muestra de adultos mayores pobres en México. Salud Publica Mex. 2011;53:26-33.

22. Fhon JR, Fabrício-Wehbe SC, Vendruscolo TR, Stackfleth R, Marques S, Rodrigues RA. Accidental falls in the elderly and their relation with functional capacity. Rev. Latino-Am. Enfermagem. 2012;20(5):927-34.

23. Shumway-Cook A, Ciol MA, Hoffman J, Dudgeon BJ, Yorkston $\mathrm{K}$, Chan L. Falls in the Medicare population: incidence, associated factors, and impact on health care. Phys Ther. 2009;89(4):324-32.

24. Leung A, Chi I, Lou VW, Chan KS. Psychosocial risk factors associated with falls among Chinese community- 
dwelling older adults in Hong Kong. Health Soc Care Commun. 2010;18(3):272-81.

25. Muir SW, Gopaul K, Montero Odasso MM. The role of cognitive impairment in fall risk among older adults: a systematic review and meta-analysis. Age Ageing. 2012;41(3):299-308.

26. Delbaere K, Close JC, Heim J, Sachdev PS, Brodaty $H$, Slavin $M J$, et al. A multifactorial approach to understanding fall risk in older people. J Am Geriatr Soc. 2010;58(9):1679-85. 